NEWS AND VIEWS

\title{
Fungal gene clusters
}

\author{
Ralph A Dean
}

\section{The genome of the fungus that causes corn smut disease has been sequenced.}

What is the molecular basis of pathogenicity? In the case of prokaryotes, our understanding of virulence was dramatically altered by the discovery of pathogenicity islands, groups of clustered genes that can undergo rapid, radical changes ${ }^{1-3}$. A recent report on the genome landscape of the biotrophic fungal pathogen Ustilago maydis reveals that a similar phenomenon may exist in eukaryotes. Writing in Nature, Kamper et al. ${ }^{4}$ show that $U$. maydis, the agent of a widespread agricultural blight known as corn smut disease, contains gene clusters that appear to share characteristics with bacterial pathogenicity islands, in that they both express secreted gene products involved in pathogenicity (Fig. 1).

Although considered a lower eukaryote, U. maydis is far from primitive and is in many respects a very sophisticated organism ${ }^{5}$. It is dimorphic, having both a haploid yeast and a dikaryotic filamentous form, and attacks corn only in its filamentous form. As a biotrophic pathogen, this fungus must keep its host alive to survive and reproduce. Thus, it appears to operate in stealth mode, suppressing or evading host defenses until late in the infection cycle. Then, as infected corn ears begin to mature, they are transformed by the pathogen into grotesque tumors containing millions of black diploid spores that erupt, leading to a dramatic smutty appearance.

Kamper et al. decoded the U. maydis genome to gain new insight into this organism's biotrophic lifestyle. The genome turns out to be smaller (20.5 Mb) than many other filamentous fungal genomes, containing $\sim 6,902$ predicted genes ${ }^{6,7}$. Consistent with its biotrophism, U. maydis lacks many signatures found in more aggressive pathogens, such as secreted hydrolytic enzymes for cell wall degradation ${ }^{8,9}$. Remarkably, nearly $20 \%$ of its 426 secreted proteins are arranged in 12 gene clusters containing 3-26 genes. Moreover, genes in seven of these clusters appear to be expressed only in tumor tissue by a known central regulator of pathogenic development.

To directly assess the role of these putative gene clusters in disease, the authors constructed deletion mutants for all 12 clusters. Although all

Ralph A. Dean is at the Department of Plant Pathology at North Carolina State University, Campus Box 7251, Raleigh, North Carolina 27695

e-mail: ralph_dean@ncsu.edu

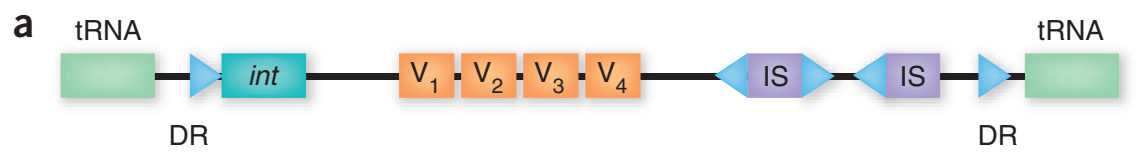

Bacterial pathogenicity island

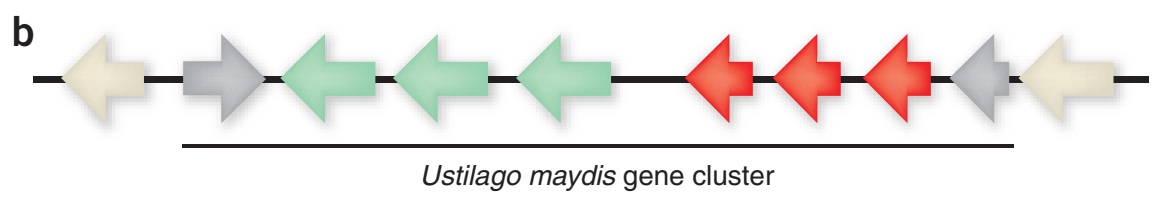

Figure 1 Schematic representation of gene clusters involved in bacterial and fungal pathogenicity. (a) Bacterial pathogenicity island. V1-V4, virulence genes; IS, remains of mobile elements; int, integrase; DR, direct repeat; tRNA, tRNA genes flanking the island. (b) Ustilago maydis 'biotrophy clusters'. White arrows, genes flanking the cluster; green and red arrows, tandem-repeated genes; gray arrows, unrelated genes.

knockout mutants appeared normal, five had an altered disease phenotype-in four of them, disease symptoms were mitigated, and in one they were exacerbated. This discovery led the authors to suggest that identification of these so-called 'biotrophy clusters' may increase understanding of fungal disease just as the discovery of pathogenicity islands did for bacterial infection.

There are several similarities between the novel clusters and pathogenicity islands. In bacteria, pathogenicity islands are flanked by tRNA or tRNA-like genes and contain a number of mobility genes, such as integrases ${ }^{2}$ (Fig. 1a). An important feature of the islands is that they differ in base composition from the rest of the genome, suggesting that they were obtained via horizontal gene transfer ${ }^{10}$.

Although the authors established that 8 of the 12 U. maydis clusters contain two to five related genes that may have arisen through duplication, their origin is unknown. Do the regions contain tRNA genes or mobile genetic elements? Like pathogenicity islands, the $U$. maydis clusters contain virulence genes. Deletion of the entire cluster affects virulence in five cases. However, is each gene in a cluster required for virulence? What about the other seven gene clusters that do not appear to be associated with virulence? The authors note that, for four of these clusters, related and perhaps redundant genes were found elsewhere in the genome. Other examples of coexpressed clustered virulence genes are well documented in fungi and include a number of toxin biosynthetic clusters and pigmentation genes $^{11,12}$. However, these gene products are not necessarily secreted, as they appear to be in the U. maydis clusters.

These findings suggest that it is probably not realistic to develop a single agent that counteracts all these virulence factors. However, as a number of common processes are involved in the secretion of virulence factors, it may be possible to target one of these processes to control fungal invasion. Gilbert et al. ${ }^{13}$ recently showed that deletion of a P-type ATPase, which is part of the exocytosis apparatus, is essential for protein secretion and pathogenicity in the rice blast fungal pathogen Magnaporthe grisea. Thus, strategies that focus on blocking P-type ATPases and other core elements required for secretion and delivery of proteins to the host may hold great promise.

1. Kim, J.F. \& Alfano, J.R. Curr. Top. Microbiol. Immunol. 264, 127-147 (2002)

2. Schmidt, H. \& Hensel, M. Clin. Microbiol. Rev. 17, 14-56 (2004).

3. Hacker, J. \& Carniel, E. EMBO Rep. 2, 376-381 (2001).

4. Kamper, J. et al. Nature 444, 97-101 (2006).

5. Garcia-Pedrajas, M.D. \& Gold, S.E. Adv. Appl. Microbiol. 56, 263-290 (2004).

6. Galagan, J.E., Henn, M.R., Ma, L.J., Cuomo, C.A. \& Birren, B. Genome Res. 15, 1620-1631 (2005).

7. Fitzpatrick, D.A., Logue, M.E., Stajich, J.E. \& Butler, G. BMC Evol. Biol. 6, 99 (2006).

8. Dean, R.A. et al. Nature 434, 980-986 (2005)

9. Sexton, A.C. \& Howlett, B.J. Eukaryot. Cell 5, 1941 1949 (2006).

10. Lesic, B. \& Carniel, E. J. Bacteriol. 187, 3352-3358 (2005).

11. Kim, J.E. et al. Appl. Environ. Microbiol. 72, 1645 1652 (2006).

12. Kimura, N. \& Tsuge, T. J. Bacteriol. 175, 4427-4435 (1993).

13. Gilbert, M.J., Thornton, C.R., Wakley, G.E. \& Talbot, N.J. Nature 440, 535-539 (2006). 